Bull. Korean Math. Soc. 52 (2015), No. 1, pp. 239-246

http://dx.doi.org/10.4134/BKMS.2015.52.1.239

\title{
QUASI-COMMUTATIVE SEMIGROUPS OF FINITE ORDER RELATED TO HAMILTONIAN GROUPS
}

\author{
Mohammad Reza Sorouhesh and Hossein Doostie
}

\begin{abstract}
If for every elements $x$ and $y$ of an associative algebraic structure $(S, \cdot)$ there exists a positive integer $r$ such that $a b=b^{r} a$, then $S$ is called quasi-commutative. Evidently, every abelian group or commutative semigroup is quasi-commutative. Also every finite Hamiltonian group that may be considered as a semigroup, is quasi-commutative however, there are quasi-commutative semigroups which are non-group and non commutative. In this paper, we provide three finitely presented noncommutative semigroups which are quasi-commutative. These are the first given concrete examples of finite semigroups of this type.
\end{abstract}

\section{Introduction}

The quasi-commutativity property in algebraic structures is one of the interesting ideas which has been studied by many authors since 1971. The classification or identification of certain major classes of semigroups has been studied as well. For more and detailed descriptions on the quasi-commutative semigroups, quasi-commutative Hamiltonian semigroups, quasi-commutative super Hamiltonian semigroups and periodic Hamiltonian semigroups one may consult the prolific articles $[3,6,8,9,10,11]$. For our purposes, we need to recall the notion of presentation $\langle X \mid R\rangle$ of formal generators $X$ and relators $R$ where, $\langle X \mid R\rangle$ is defined properly for finitely generated semigroups or for finitely generated monoids. Note that, $R$ is called the set of relations when $\langle X \mid R\rangle$ is a group presentation. Furthermore, some preliminaries and more information on the semigroups and monoids presentation are required in the presentation theory of semigroups which may be found in $[4,5,7]$. The natural question which may be posed here is how the non-group quasi-commutative semigroups may be constructed? In this paper, we construct three finitely presented non-commutative semigroups which are not groups and show that they are finite and quasi-commutative. This construction is based on examining possible semigroup presentations of the known quasi-commutative finite

Received December 21, 2013; Revised January 23, 2014.

2010 Mathematics Subject Classification. 20M05.

Key words and phrases. quasi-commutativity, finitely presented semigroups. 
groups. Our notation is fairly standard and we follow the celebrated references Howie [4] and Clifford [7] for the basic notions. Consider the presentations

$$
\begin{aligned}
& \pi_{1}=\left\langle a, b \mid a^{5}=a, b^{2}=a^{2}, b a=a b^{3}\right\rangle, \\
& \pi_{2}=\left\langle a, b, c_{1}, c_{2}, \ldots, c_{k}\right| a^{5}=a, b^{2}=a^{2}, b a=a b^{3}, c_{i}^{3}=c_{i}, a c_{i}=c_{i} a, \\
& \left.\quad b c_{i}=c_{i} b, c_{i} c_{j}=c_{j} c_{i}, 1 \leq i, j \leq k\right\rangle, \\
& \pi_{3}=\left\langle a, b, c_{1}, c_{2}, \ldots, c_{k}, d\right| a^{5}=a, b^{2}=a^{2}, b a=a b^{3}, c_{i}^{3}=c_{i}, d^{p+1}=d, \\
& \left.\quad a c_{i}=c_{i} a, d a=a d, d b=b d, d c_{i}=c_{i} d, b c_{i}=c_{i} b, c_{i} c_{j}=c_{j} c_{i}, 1 \leq i, j \leq k\right\rangle,
\end{aligned}
$$

where $p$ is an odd prime and let $S g(\pi)$ and $G p(\pi)$ be the semigroup and the group presented by $\pi$, respectively. For the detailed information on the semigroup presentation, one may consult Campbell et al. [1, 2]. The semigroup $S g\left(\pi_{1}\right)$ is of order 9 and may be considered as the smallest non-group example of quasi-commutative semigroup. We'll give a short proof for this, in Section 2 after the investigation of preliminary properties of a non-commutative quasicommutativity. However, our main results are the following propositions:

Proposition A. $S g\left(\pi_{2}\right)$ is a finite quasi-commutative semigroup of order

$$
10 \times 3^{k}-1 .
$$

Proposition B. $S g\left(\pi_{3}\right)$ is a finite quasi-commutative semigroup of order

$$
10\left(3^{k}-1\right) p+10 \times 3^{k}+10 p-1 .
$$

\section{Preliminary properties}

A semigroup $S$ is called quasi-commutative if for every $x, y \in S$ there exists a positive integer $r$ such that $x y=y^{r} x$. Following Mukherjee [8] we first recall certain preliminary properties of quasi-commutative semigroups. For two elements $a, b$ of a semigroup $S$ if $a b=b^{r} a$ holds for some positive integers $r$ then, the following identities hold for every positive integer $k$ :

$$
\begin{aligned}
a b^{k} & =b^{r k} a, a^{k} b=b^{r^{k}} a^{k}, \\
(a b)^{k} & =b^{r+r^{2}+\cdots+r^{k}} \cdot a^{k},(b a)^{k}=b^{1+r+\cdots+r^{k-1}} \cdot a^{k} .
\end{aligned}
$$

Lemma 2.1. The relator $a b=b^{3} a$ holds in $S g\left(\pi_{1}\right)$. Moreover, for all values of positive integers $i$ and $k, b^{k} a^{i}=a^{i} b^{3^{i} k}$

Proof. The relators of $S g\left(\pi_{1}\right)$ give us:

$$
\begin{aligned}
a b & =a^{5} b=\left(a^{4}\right)(a b)=b^{4}(a b)=b^{3}(b a b)=b^{3}\left(a b^{3}\right) b \quad\left(\text { for } b a=a b^{3}\right) \\
& =b^{3} a b^{4}=b^{3} a a^{4}=b^{3} a^{5}=b^{3} a .
\end{aligned}
$$

To prove the last part, for a fixed $k$, we use an induction method on $i$. Also, to shorten the proof and avoid excessive exposition, we consider two cases for $i$ (odd and even) and observe that $3^{i} \equiv \pm 1(\bmod 4)$ while, $i$ is even or odd, respectively. 
Lemma 2.2. For all positive integers $k, i$ and $t$,

$$
\left(a^{i} b^{k}\right)^{t}=a^{t i} b^{k\left(1+3^{i}+3^{2 i}+\cdots+3^{(t-1) i}\right)} .
$$

Proof. For $t=1$ there is nothing to prove and using the induction method on $t$ we get:

$$
\begin{aligned}
\left(a^{i} b^{k}\right)^{t+1} & =\left(a^{i} b^{k}\right)^{t}\left(a^{i} b^{k}\right) \\
& =\left(a^{t i} b^{k\left(1+3^{i}+3^{2 i}+\cdots+3^{(t-1) i}\right)}\right)\left(a^{i} b^{k}\right) \quad \text { (by the inductive hypothesis) } \\
& =a^{t i}\left(b^{k\left(1+3^{i}+3^{2 i}+\cdots+3^{(t-1) i}\right)} a^{i}\right) b^{k} .
\end{aligned}
$$

Now, Lemma 2.1 gives us:

$$
b^{k\left(1+3^{i}+3^{2 i}+\cdots+3^{(t-1) i}\right)} a^{i}=a^{i} b^{3^{i} \times k\left(1+3^{i}+3^{2 i}+\cdots+3^{(t-1) i}\right)} .
$$

Consequently,

$$
\left(a^{i} b^{k}\right)^{t+1}=a^{t i}\left(a^{i} b^{3^{i} \times k\left(1+3^{i}+3^{2 i}+\cdots+3^{(t-1) i}\right)}\right) b^{k}=a^{(t+1) i} b^{k\left(1+3^{i}+3^{2 i}+\cdots+3^{t i}\right)} .
$$

As a result of this lemma, we get the following results which we believe that they are the key in our purposes of this section.

Lemma 2.3. The equality $\left(a^{i} b\right)^{3}=a^{i} b^{3}$ holds in $S g\left(\pi_{1}\right)$ for every positive integer $i$ and the equations $\left(a^{i} b\right)^{3}=\left(a^{i+2} b\right)=a^{3 i} b$ hold in $S g\left(\pi_{1}\right)$ for odd values of $i$. Moreover, $S g\left(\pi_{1}\right)$ is quasi-commutative.

Proof. The first two parts may be deduced by using Lemma 2.2 and by considering the relators of $S g\left(\pi_{1}\right)$. Again, using the result of Lemma 2.1 we get:

$$
S g\left(\pi_{1}\right)=\{b\} \cup\left\{a^{i}\right\} \cup\left\{a^{j} b\right\},
$$

where $1 \leq i, j \leq 4$. So, $S g\left(\pi_{1}\right)$ is of order 9 . To prove the quasi-commutativity of $S g\left(\pi_{1}\right)$, we consider different cases for $x, y \in S g\left(\pi_{1}\right)$. Obviously in the following cases we have nothing to do:

- $x=y$.

- $x, y \in\left\{a^{i} \mid 1 \leq i \leq 4\right\}$.

- $x=a^{i}, y=a^{\bar{j}} b, 1 \leq i, j \leq 4$.

- $x=a^{j} b, y=a^{i} b, 1 \leq i, j \leq 4$.

The essential cases for $x$ and $y$, that may be lead to finding appropriate integers $r, n$ satisfying $x y=y^{r} x$ and $y x=x^{n} y$ are as follows.

Case 1. $x=a^{i}$ and $y=b$. By using (A.1), we get $x y=a^{i} b=b^{3^{i}} a^{i}=(b)^{3^{i}} a^{i}$ and so, $x y=y^{\mathbf{3}^{\mathbf{i}}} x$. For even values of $i$ we may consider Lemma 2.1 to get $y x=b a^{i}=a^{i} b=x y$. And if $i$ be odd, then by Lemma 2.3 we get $y x=b a^{i}=$ $a^{i+2} b=a^{3 i} b=x^{3} y$.

Case 2. $x=a^{j} b$ and $y=b$. To calculate $x y$ we again consider (A.1) then for even values of $j$ we get $x y=\left(a^{j} b\right) b=\left(b a^{j}\right) b=b\left(a^{j} b\right)=y x$. For the odd 
values of $j$, we get:

$$
\left\{\begin{array}{l}
x y=\left(a^{j} b\right) b=\left(b^{3} a^{j}\right) b=b^{3}\left(a^{j} b\right)=y^{3} x \quad\left(\text { for }\left(a^{j} b\right) b=\left(b^{3^{j}} a^{j}\right) b\right) \\
y x=b\left(a^{j} b\right)=\left(b a^{j}\right) b=\left(a^{j+2} b\right) b=\left(a^{j} b\right)^{3} b=x^{3} y \quad\left(\text { for } b a^{j}=a^{j+2} b\right) .
\end{array}\right.
$$

\section{Proof of Proposition A}

To identify the elements of $S g\left(\pi_{2}\right)$ first we consider the following key lemma.

Lemma 3.1. The semigroup $S g\left(\pi_{2}\right)$ may be partitioned as

$S g\left(\pi_{2}\right)=\{b\} \cup\left\{a^{i}\right\} \cup\left\{a^{j} b\right\} \cup\left\{\prod_{t=1}^{h} c_{t}^{r_{h}}\right\} \cup\left\{b \prod_{t=1}^{s} c_{t}^{r_{s}}\right\} \cup\left\{a^{l} \prod_{t=1}^{p} c_{t}^{r_{p}}\right\} \cup\left\{a^{m} b \prod_{t=1}^{q} c_{t}^{r_{q}}\right\}$

where, $1 \leq h, s, p, q \leq k, 1 \leq r_{h}, r_{s}, r_{p}, r_{q} \leq 2,1 \leq i, j, m, l \leq 4$.

Proof. In light of Lemma 2.1 and the relators of $S g\left(\pi_{2}\right)$ we conclude that there is no word in this semigroup starting with $b$ or $\prod_{t=1}^{h} c_{t}^{r_{h}}$ except the words $b$ and $\prod_{t=1}^{h} c_{t}^{r_{h}}$ where, $h=1, \ldots, k, 1 \leq r_{h} \leq 2$.

Proof of Proposition A. We proceed in the same way as in Lemma 2.3. In fact, to find a proper $r$ such that $x y=y^{r} x, x, y \in S g\left(\pi_{2}\right)$ we can consider different cases for $x$ and $y$. Obviously, for the cases in which $x=y$, or

- $x=a^{i}, y=a^{j}$.

- $x=\prod_{t=1}^{h} c_{t}^{r_{h}}, y=\prod_{t=1}^{s} c_{t}^{r_{s}}$.

- $x=b \prod_{t=1}^{s} c_{t}^{r_{s}}, y=b \prod_{t=1}^{p} c_{t}^{r_{p}}$.

- $x=a^{j} \prod_{t=1}^{q} c_{t}^{r_{q}}, y=a^{l} \prod_{t=1}^{p} c_{t}^{r_{p}}$.

the desired $r$ would be 1, i.e., $x y=y x$ and Lemma 2.3 gives us $r=1$ or $r=3$ in the following cases

- $x=a^{i}, y=a^{j} b \prod_{t=1}^{h} c_{t}^{r_{h}}$.

- $x=a^{i} b, y=a^{j} \prod_{t=1}^{h} c_{t}^{r_{h}}$.

- $x=a^{i} b, y=a^{j} b \prod_{t=1}^{h} c_{t}^{r_{h}}$.

- $x=a^{i} \prod_{t=1}^{h} c_{t}^{r_{h}}, y=a^{j} b \prod_{t=1}^{s} c_{t}^{r_{s}}$.

- $x=a^{i} b \prod_{t=1}^{h} c_{t}^{r_{h}}, y=a^{j} b \prod_{t=1}^{s} c_{t}^{r_{s}}$

Moreover, regarding the relators of $S g\left(\pi_{2}\right)$, the pair of words as

- $x=a^{i} b, y=\prod_{t=1}^{h} c_{t}^{r_{h}}$.

- $x=\prod_{t=1}^{p} c_{t}^{r_{p}}, y \in\left\{b \prod_{t=1}^{s} c_{t}^{r_{s}}\right\} \cup\left\{a^{l} \prod_{t=1}^{p} c_{t}^{r_{p}}\right\}$.

- $x=b, y \in\left\{\prod_{t=1}^{h} c_{t}^{r_{h}}\right\} \cup\left\{b \prod_{t=1}^{s} c_{t}^{r_{s}}\right\}$.

- $x=a^{i}, y \in\left\{\prod_{t=1}^{h} c_{t}^{r_{h}}\right\} \cup\left\{a^{l} \prod_{t=1}^{p} c_{t}^{r_{p}}\right\}$.

- $x=a^{m} b \prod_{t=1}^{q} c_{t}^{r_{q}}, y=\prod_{t=1}^{h} c_{t}^{r_{h}}$.

are commute. So, for each such pair we get $x y=y x$. Summarizing all other possible cases for $x$ and $y$, there are exactly six cases. To shorten the proof, we give the proof of quasi-commutativity of the pairs $(x, y)$ and $(y, x)$ just in the cases 1, 4 and 5 . These six cases are: 
- $x=a^{i}, y=b \prod_{t=1}^{h} c_{t}^{r_{h}}$.

- $x=a^{i} b, y=b \prod_{t=1}^{h} c_{t}^{r_{h}}$.

- $x=b, y=a^{j} \prod_{t=1}^{h} c_{t}^{r_{h}}$.

- $x=b, y=a^{j} b \prod_{t=1}^{h} c_{t}^{r_{h}}$.

- $x=a^{i} \prod_{t=1}^{h} c_{t}^{r_{h}}, y=b \prod_{t=1}^{s} c_{t}^{r_{s}}$.

- $x=b \prod_{t=1}^{h} c_{t}^{r_{h}}, y=a^{j} b \prod_{t=1}^{s} c_{t}^{r_{s}}$.

In the first case by (A.1) and the Lemma 2.1 we get $x y=\left(b^{3^{i}} a^{i}\right)\left(\prod_{t=1}^{h} c_{t}^{r_{h}}\right)$ and $y x=\left(b a^{i}\right)\left(\prod_{t=1}^{h} c_{t}^{r_{h}}\right)$. If $i$ is even so according to the relators of $S g\left(\pi_{2}\right)$ we get $x y=y x$ and if $i$ is odd then,

$$
\begin{aligned}
& x y=\left(b^{3} \prod_{t=1}^{h} c_{t}^{r_{h}}\right) a^{i}=\left(b \prod_{t=1}^{h} c_{t}^{r_{h}}\right)^{3} a^{i}=y^{\mathbf{3} x}, \\
& y x=\left(a^{i+2} b\right)\left(\prod_{t=1}^{h} c_{t}^{r_{h}}\right)=a^{3 i}\left(b \prod_{t=1}^{h} c_{t}^{r_{h}}\right)=x^{\mathbf{3}} y .
\end{aligned}
$$

In the fourth case by (A.1) we have $x y=b\left(a^{j} b \prod_{t=1}^{h} c_{t}^{r_{h}}\right)=\left(b a^{j}\right)\left(\prod_{t=1}^{h} c_{t}^{r_{h}} b\right)$ and $y x=\left(a^{j} b \prod_{t=1}^{h} c_{t}^{r_{h}}\right) b=\left(a^{j} b\right)\left(b \prod_{t=1}^{h} c_{t}^{r_{h}}\right)=\left(b^{3^{j}} a^{j}\right)\left(b \prod_{t=1}^{h} c_{t}^{r_{h}}\right)$. Suppose that $j$ is even so $x y=\left(a^{j} b\right)\left(\prod_{t=1}^{h} c_{t}^{r_{h}} b\right)=\left(a^{j} b \prod_{t=1}^{h} c_{t}^{r_{h}}\right) b=y x$. And if $j$ is odd so according to Lemmas 2.1, 2.3 and the 4 th relator we get

$$
\begin{aligned}
x y & =\left(a^{j+2} b\right)\left(\prod_{t=1}^{h} c_{t}^{r_{h}} b\right)=\left(a^{3 j} b \prod_{t=1}^{h} c_{t}^{r_{h}}\right) b \\
& =\left(a^{3 j} b^{5}\right)\left(\prod_{t=1}^{h} c_{t}^{3 r_{h}} b\right)=\left(a^{j} b \prod_{t=1}^{h} c_{t}^{r_{h}}\right)^{3} b=y^{\mathbf{3}} x, \\
y x & =\left(b^{3} a^{j}\right)\left(b \prod_{t=1}^{h} c_{t}^{r_{h}}\right)=\left(a^{j} b^{3^{1+j}}\right)\left(b \prod_{t=1}^{h} c_{t}^{r_{h}}\right) \\
& =\left(a^{j} b \prod_{t=1}^{h} c_{t}^{r_{h}}\right) b=x y .
\end{aligned}
$$

In the fifth case regarding to the 5th and 6 th relators of the semigroup and (A.1), we have

$$
\begin{aligned}
x y & =\left(a^{i} \prod_{t=1}^{h} c_{t}^{r_{h}}\right)\left(b \prod_{t=1}^{s} c_{t}^{r_{s}}\right)=\left(a^{i} b\right)\left(\prod_{t=1}^{s} c_{t}^{r_{s}} \prod_{t=1}^{h} c_{t}^{r_{h}}\right) \\
& =\left(b^{3^{i}} \prod_{t=1}^{s} c_{t}^{r_{s}}\right)\left(a^{i} \prod_{t=1}^{h} c_{t}^{r_{h}}\right), \\
y x & =\left(b \prod_{t=1}^{s} c_{t}^{r_{s}}\right)\left(a^{i} \prod_{t=1}^{h} c_{t}^{r_{h}}\right)=\left(\prod_{t=1}^{h} c_{t}^{r_{h}} \prod_{t=1}^{s} c_{t}^{r_{s}}\right)\left(b a^{i}\right) \\
& =\left(\prod_{t=1}^{h} c_{t}^{r_{h}} \prod_{t=1}^{s} c_{t}^{r_{s}}\right)\left(a^{i} b^{3^{i}}\right)=\left(a^{i} \prod_{t=1}^{h} c_{t}^{r_{h}}\right)\left(b^{3^{i}} \prod_{t=1}^{s} c_{t}^{r_{s}}\right) .
\end{aligned}
$$


Now if $i$ is even so $x y=\left(b \prod_{t=1}^{s} c_{t}^{r_{s}}\right)\left(a^{i} \prod_{t=1}^{h} c_{t}^{r_{h}}\right)=y x$. And if $i$ is odd so Lemma 2.2 and the 4 th, 5 th and 6 th relators of $S g\left(\pi_{2}\right)$ give us

$$
\begin{aligned}
x y & =\left(b^{3} \prod_{t=1}^{s} c_{t}^{r_{s}}\right)\left(a^{i} \prod_{t=1}^{h} c_{t}^{r_{h}}\right)=\left(b^{3} \prod_{t=1}^{s} c_{t}^{3 r_{s}}\right)\left(a^{i} \prod_{t=1}^{h} c_{t}^{r_{h}}\right) \\
& =\left(b \prod_{t=1}^{s} c_{t}^{r_{s}}\right)^{3}\left(a^{i} \prod_{t=1}^{h} c_{t}^{r_{h}}\right)=y^{\mathbf{3}} x, \\
y x & =\left(a^{i} \prod_{t=1}^{h} c_{t}^{r_{h}}\right)\left(b^{3} \prod_{t=1}^{s} c_{t}^{r_{s}}\right)=\left(a^{i} \prod_{t=1}^{h} c_{t}^{r_{h}} b^{2}\right)\left(b \prod_{t=1}^{s} c_{t}^{r_{s}}\right) \\
& =\left(a^{i+2} \prod_{t=1}^{h} c_{t}^{r_{h}}\right)\left(b \prod_{t=1}^{s} c_{t}^{r_{s}}\right)=\left(a^{3 i} \prod_{t=1}^{h} c_{t}^{3 r_{h}}\right)\left(b \prod_{t=1}^{s} c_{t}^{r_{s}}\right) \\
& =\left(a^{i} \prod_{t=1}^{h} c_{t}^{r_{h}}\right)^{3}\left(b \prod_{t=1}^{s} c_{t}^{r_{s}}\right)=x^{\mathbf{3}} y .
\end{aligned}
$$

A quick use of Lemma 3.1 gives us the order of $S g\left(\pi_{2}\right)$ and this completes the proof.

\section{Proof of Proposition B}

Proof of Proposition B. A similar method to that of Proposition A may be used here to investigating the quasi-commutating of the semigroup $S g\left(\pi_{3}\right)$. This will be performed by considering all cases for the pair $(x, y)$ of $S g\left(\pi_{3}\right)$ by the relators of this semigroup. We recognize all of these cases and omit the proof, which is similar to that of the last section. Assuming

$$
1 \leq i, j \leq 4,1 \leq h \leq k, 1 \leq r_{h}, r_{s} \leq 2,1 \leq m, n \leq p
$$

the possible cases are:

- $x=b d^{m}, y \in\left\{\begin{array}{l}a^{i} d^{n}, a^{i} \prod_{t=1}^{h} c_{t}^{r_{h}} d^{n}, a^{i} b \prod_{t=1}^{h} c_{t}^{r_{h}} d^{n}, a^{i} \\ a^{i} b \prod_{t=1}^{h} c_{t}^{r_{h}}, a^{i} b d^{n}, a^{i} \prod_{t=1}^{h} c_{t}^{r_{h}}\end{array}\right.$

- $x=a^{i} d^{m}, y \in\left\{\begin{array}{l}a^{j} \prod_{t=1}^{h} c_{t}^{r_{h}}, b \prod_{t=1}^{h} c_{t}^{r_{h}}, b, a^{j} b \prod_{t=1}^{h} c_{t}^{r_{h}} \\ b \prod_{t=1}^{h} c_{t}^{r_{h}} d^{n}, b d^{n}, a^{j} b \prod_{t=1}^{h} c_{t}^{r_{h}} d^{n}, a^{j} b d^{n}\end{array}\right.$

- $x=a^{i} \prod_{t=1}^{h} c_{t}^{r_{h}} d^{m}, y \in\left\{\begin{array}{l}b, b \prod_{t=1}^{h} c_{t}^{r_{s}}, a^{j} b \prod_{t=1}^{h} c_{t}^{r_{s}}, b d^{n}, d^{n} \\ b \prod_{t=1}^{h} c_{t}^{r_{s}} d^{n}, a^{j} b \prod_{t=1}^{h} c_{t}^{r_{s}} d^{n}, a^{j} b d^{n}\end{array}\right.$

- $x=b \prod_{t=1}^{h} c_{t}^{r_{h}} d^{m}, y \in\left\{\begin{array}{l}a^{i}, a^{i} \prod_{t=1}^{h} c_{t}^{r_{s}}, a^{i} b \prod_{t=1}^{h} c_{t}^{r_{s}}, a^{i} \prod_{t=1}^{h} c_{t}^{r_{s}} d^{n} \\ a^{i} b \prod_{t=1}^{h} c_{t}^{r_{s}} d^{n}, a^{i} b d^{n}, a^{i} d^{n}\end{array}\right.$

- $x=a^{i} b \prod_{t=1}^{h} c_{t}^{r_{h}} d^{m}, y \in\left\{\begin{array}{l}b, a^{j}, a^{j} \prod_{t=1}^{h} c_{t}^{r_{s}}, b \prod_{t=1}^{h} c_{t}^{r_{s}}, a^{j} b \prod_{t=1}^{h} c_{t}^{r_{s}} \\ b d^{n}, a^{j} d^{n}, a^{j} \prod_{t=1}^{h} c_{t}^{r_{s}} d^{n}, b \prod_{t=1}^{h} c_{t}^{r_{s}} d^{n} \\ a^{j} b \prod_{t=1}^{h} c_{t}^{r_{s}} d^{n}, a^{j} b d^{n}\end{array}\right.$

- $x \in\left\{b, b \prod_{t=1}^{h} c_{t}^{r_{h}}\right\}, y \in\left\{\begin{array}{l}a^{i}, a^{i} \prod_{t=1}^{h} c_{t}^{r_{h}}, a^{i} b \prod_{t=1}^{h} c_{t}^{r_{h}}, a^{i} d^{m} \\ a^{i} \prod_{t=1}^{h} c_{t}^{r_{h}} d^{m}, a^{i} b \prod_{t=1}^{h} c_{t}^{r_{h}} d^{m}, a^{i} b d^{n}\end{array}\right.$ 
- $x=\left\{a^{i}, a^{i} \prod_{t=1}^{h} c_{t}^{r_{h}}\right\}, y \in\left\{\begin{array}{l}b, b \prod_{t=1}^{h} c_{t}^{r_{h}}, a^{j} b \prod_{t=1}^{h} c_{t}^{r_{h}}, b d^{m} \\ b \prod_{t=1}^{h} c_{t}^{r_{h}} d^{m}, a^{j} b \prod_{t=1}^{h} c_{t}^{r_{h}} d^{m}, a^{j} b d^{n}\end{array}\right.$

- $x=a^{i} b \prod_{t=1}^{h} c_{t}^{r_{h}}, y \in\left\{\begin{array}{l}b, a^{j}, a^{j} \prod_{t=1}^{h} c_{t}^{r_{s}}, b \prod_{t=1}^{h} c_{t}^{r_{s}}, a^{j} b \prod_{t=1}^{h} c_{t}^{r_{s}} \\ b d^{m}, b \prod_{t=1}^{h} c_{t}^{r_{s}} d^{m}, a^{j} b \prod_{t=1}^{h} c_{t}^{r_{s}} d^{m}, a^{j} b d^{n}\end{array}\right.$

- $x=a^{i} b d^{m}, y \in\left\{\begin{array}{l}b, a^{j}, a^{j} \prod_{t=1}^{h} c_{t}^{r_{s}}, b \prod_{t=1}^{h} c_{t}^{r_{s}}, a^{j} b \prod_{t=1}^{h} c_{t}^{r_{s}} \\ a^{j} b \prod_{t=1}^{h} c_{t}^{r_{s}} d^{n}, b d^{n}, a^{i} d^{n}, b \prod_{t=1}^{h} c_{t}^{s_{s}} d^{m} \\ a^{j} \prod_{t=1}^{h} c_{t}^{r_{s}} d^{n}, b \prod_{t=1}^{h} c_{t}^{r_{s}} d^{n}, a^{j} b d^{n}\end{array}\right.$

To complete the proof of Proposition B, one may use the following partition of,

$$
\begin{aligned}
S g\left(\pi_{3}\right)= & \left\{a^{i} b d^{m}\right\} \cup\{b\} \cup\left\{a^{i}\right\} \cup\left\{a^{i} b \prod_{t=1}^{h} c_{t}^{r_{h}}\right\} \cup\left\{b \prod_{t=1}^{h} c_{t}^{r_{h}}\right\} \cup\left\{a^{i} \prod_{t=1}^{h} c_{t}^{r_{h}}\right\} \\
& \cup\left\{a^{i} b \prod_{t=1}^{h} c_{t}^{r_{h}} d^{m}\right\} \cup\left\{b \prod_{t=1}^{h} c_{t}^{r_{h}} d^{m}\right\} \cup\left\{a^{i} \prod_{t=1}^{h} c_{t}^{r_{h}} d^{m}\right\} \cup\left\{\prod_{t=1}^{h} c_{t}^{r_{h}} d^{m}\right\} \\
& \cup\left\{a^{i} d^{m}\right\} \cup\left\{b d^{m}\right\} \cup\left\{a^{i} b\right\} \cup\left\{\prod_{t=1}^{h} c_{t}^{r_{h}}\right\} \cup\left\{d^{m}\right\} .
\end{aligned}
$$

So,

$$
\left|S g\left(\pi_{3}\right)\right|=10\left(3^{k}-1\right) p+10 \times 3^{k}+10 p-1 .
$$

Acknowledgement. The authors would like to express their appreciation of the reviewers' excellent comments in improving this paper.

\section{References}

[1] C. M. Campbell, E. F. Robertson, N. Ruškuc, and R. M. Thomas, Semigroup and group presentations, Bull. Lond. Math. Soc. 27 (1995), no. 1, 46-50.

[2] C. M. Campbell, E. F. Robertson, N. Ruškuc, R. M. Thomas, and Y. Ünlü, Certain one-relator products of semigroups, Comm. Algebra 23 (1995), no. 14, 5207-5219.

[3] M. Chacron and G. Thierrin, $\sigma$-reflexive semigroups and rings, Canad. Math. Bull. 15 (1972), 185-188.

[4] A. H. Clifford and G. B. Preston, The Algebraic Theory of Semigroups I, Amer. Math. Soc., 1961.

[5] A. C. Spoletini and A. Varisco, Quasicommutative semigroups and $\sigma$-reflexive semigroups, Semigroup Forum 19 (1980), no. 4, 313-321.

[6] — Quasi Hamiltonian semigroups, Czechoslovak Math. J. 33 (1983), no. 1, 131140.

[7] J. M. Howie, An Introduction to Semigroup Theory, Academic Press Inc., 1976.

[8] N. P. Mukherjee, Quasicommutative semigroups. I, Czechoslovak Math. J. 22 (1972), 449-453.

[9] B. Ponděliček, Note on Quasi-Hamiltonian semigroups, Časopis pro pěstování matematiky 110 (1985), no. 4, 356-358.

[10] K. P. Shum and X. M. Ren, On super Hamiltonian semigroups, Czechoslovak Math. J. 54 (2004), no. 1, 247-252. 
[11] K. P. Shum and L. Zhang, Generalized Quasi Hamiltonian semigroups, Int. J. Pure Appl. Math. 53 (2009), no. 4, 461-475.

Mohammad Reza Sorouhesh

Department of Mathematics

Tehran Science and Research Branch Islamic Azad University

TEHran, 14515/1775, IrAN

E-mail address: sorouhesh@azad.ac.ir

Hossein Doostie

Department of Mathematics

Tehran Science and Research Branch Islamic Azad University

TEHRAN, 14515/1775, IRAN

E-mail address: doostih@gmail.com 